typed norms and behaviours have been associated with deleterious health outcomes, and among adult men, endorsement of certain masculine norms (such as self-reliance) has been associated with suicidal ideation and poorer mental health. While the examination of masculinity is a useful means of understanding health risks in men and boys, there has been little quantitative examination of this relationship, particularly among adolescents. This study aimed to examine associations between endorsement of masculine norms and suicidal ideation in a representative sample of Australian adolescents.

Methods Using data from the Australian Longitudinal Study on Male Health, this study examined associations between specific masculine constructs and suicidal ideation among 826 Australian boys/young men aged 15-18 years at baseline. Masculine norms were measured in Wave 1, using the Conformity to Masculine Norms Inventory (CMNI-22). Suicidal ideation was a single item from the Patient Health Questionnaire (PHQ9), and was measured in Wave 2 (when participants were 17-20 years of age). Logistic regression analysis was conducted, adjusting for available confounders.

Results Results showed that after adjustment for parental education and area socio-economic position, greater conformity to violent norms (OR 1.23, 95\%CI: 1.03-1.47) and self-reliance norms (OR 1.49, 95\%CI: 1.15-1.70) were associated with higher risk of suicidal ideation. Greater conformity to norms regarding heterosexuality was associated with reduced risk of suicidal ideation (OR 0.80, 95\%CI: 0.68-0.91).

Conclusion Among the adolescent males in this sample, we found that high conformity to norms of violence and self-reliance was associated with greater risk of suicidal ideation, while high conformity to norms of heterosexuality was associated with reduced suicidal ideation. Such results do not indicate that being heterosexual is protective but highlight the broader buffering effect of conforming to the masculine norm of heterosexuality. Maximising adolescent health is recognized as key to a sustainable, healthy and equitable future. These results suggest that conforming to certain masculine norms may be deleterious for adolescent male health and highlight the importance of presenting multiple ways of being a male. This is vital in shifting social norms toward a society that supports various, and varying forms of masculinity, particularly in terms of sexuality.

\section{OP58 THE ROLE OF FAMILY AND PEER SUPPORT IN PROMOTING RESILIENCE IN THE FACE OF BULLYING AND CYBERBULLYING: EVIDENCE FROM SCHOOL CHILDREN IN SCOTLAND}

${ }^{1}$ RJ Shaw*, ${ }^{2}$ DB Currie, ${ }^{1} \mathrm{G}$ Smith, ${ }^{3} \mathrm{JC}$ Inchley, ${ }^{1} \mathrm{DJ}$ Smith. ${ }^{1}$ Institute of Health and Wellbeing, University of Glasgow, Glasgow, UK; ${ }^{2}$ School of Medicine, University of St Andrews, St Andrews, UK; ${ }^{3}$ MRC/CSO Social and Public Health Sciences Unit, University of Glasgow, Glasgow, UK

\subsection{6/jech-2019-SSMabstracts.59}

Background Being bullied is a threat to children's mental wellbeing but there is some evidence that emotional support may buffer children against bullying victimisation. Modern technology has changed the way that children interact, such that cyberbullying is now an additional bullying exposure. We investigated whether bullying and cyberbullying victimisation posed similar threats and whether family and peer support promoted resilience to adverse wellbeing as an outcome.
Methods Data were collected on 5,286 children in grades P7 (Age 11), S2 (Age 13), and S4 (Age 15) from 208 Schools in the 2017/18 Health Behaviour in School Aged Children in Scotland Survey. Resilience was operationalised with statistical interactions between self-reported measures of bullying or cyberbullying victimisation (None/Occasional/Frequent) and family or peer support (Low/Intermediate/High) in the prediction of wellbeing (WHO-5 Wellbeing Index). Analyses were carried out separately by gender with multilevel models, adjusted for sociodemographic factors and school grade, used to generate predictive margins.

Results $14.2 \%$ of children were frequently bullied whereas $4.6 \%$ were frequently cyberbullied, with $3.1 \%$ being both frequently bullied and cyberbullied. Both bullying and cyberbullying had negative relationships with wellbeing. The relationship between cyberbullying and wellbeing was stronger, especially for children who were victims of cyberbullying but not traditional bullying. For example, the adjusted predicted mean WHO-5 scores for girls were as follows: never bullied 58.8 (95\%CI 57.6-60.0), frequently bullied but not cyberbullied 50.5(95\%CI 46.8-54.1) frequently both bullied and cyberbullied 40.5(95\%CI 36.2-44.7), and frequently cyberbullied but not bullied 36.2(95\%CI 23.1-49.5).

Family support was associated with resilience to the consequences of both bullying and cyberbullying victimisation. For example, among never bullied girls, high family support had a modest relationship with wellbeing, the adjusted predicted mean WHO-5 score was 63.1 (95\%CI 61.7-64.4) for girls with high support and 57.4 (95\%CI 54.8-60.1) for girls with low support. For frequently bullied girls, family support was much more strongly associated with wellbeing, the adjusted predicted mean WHO-5 score for girls with high support was $56.0(95 \%$ CI $52.4-59.6)$ compared to $38.3(95 \%$ CI $31.8-44.8$ ) for those with low family support. Results for boys were similar but the protective associations of family support were reduced. There was no evidence that support from friends was associated with resilience to bullying or cyberbullying.

Conclusion Cyberbullying is not as common as bullying but may pose a greater threat to adolescent wellbeing. Supportive families are associated with resilience among victims of both bullying and cyberbullying.

\section{OP59 ARTS ENGAGEMENT AND SELF-ESTEEM IN CHILDREN: RESULTS FROM A PROPENSITY SCORE MATCHING ANALYSIS}

D Fancourt, HW Mak*, D Fancourt. Behavioural Science and Health, University College London, London, UK

\subsection{6/jech-2019-SSMabstracts.60}

Background Self-esteem is regarded as vital to children's social and cognitive development and emotional well-being. To date, a few studies have suggested that arts activities can improve self-esteem in young people. However, such studies have mainly focused on small samples. This research therefore used a UK national, representative study, Millennium Cohort Study (MCS), to explore the relationship between arts engagement and children's self-esteem. Given that arts engagement is socially patterned, propensity score matching (PSM) was implemented for analysis.

Methods This study analysed data from the MCS Sweep 5 interviews when respondents were aged 11 ( $\mathrm{N}=6209)$. 